\title{
Differentiated regulation of immune-response related genes between LUAD and LUSC subtypes of lung cancers
}

\author{
Mengzhu Chen ${ }^{1}$, Xiuying Liu ${ }^{2}$, Jie Du ${ }^{3}$, Xiu-Jie Wang ${ }^{2}$, Lixin Xia ${ }^{1}$ \\ ${ }^{1}$ State Key Laboratory of Respiratory Disease for Allergy at Shenzhen University, Department of Biochemistry and Molecular \\ Biology, Health Science Center of Shenzhen University, School of Medicine, Shenzhen University, Guangdong 518060, China \\ ${ }^{2}$ Key Laboratory of Genetic Network Biology, Collaborative Innovation Center of Genetics and Development, Institute of \\ Genetics and Developmental Biology, Chinese Academy of Sciences, Beijing 100101, China \\ ${ }^{3}$ Beijing Anzhen Hospital, Capital Medical University, Beijing Collaborative Innovative Center for Cardiovascular Disorders, \\ The Key Laboratory of Remodeling-Related Cardiovascular Diseases, Ministry of Education, Beijing Institute of Heart Lung \\ and Blood Vessel Diseases, Beijing 100029, China
}

Correspondence to: Xiu-Jie Wang, email: xjwang@genetics.ac.cn

Lixin Xia, email: xialixin@szu.edu.cn

Keywords: LUAD, LUSC, immune-response related genes, expression features, cancer progression rate

Received: August 23, 2016

Accepted: October 29, 2016

Published: November 15, 2016

\section{ABSTRACT}

Lung squamous cell carcinoma (LUSC) and lung adenocarcinoma (LUAD) are the two major subtypes of lung cancer, with LUSC exhibits faster progression rate than LUAD. To investigate the roles of immune-response related genes (IRGs) in lung cancer progression, we used LUAD and LUSC samples at different cancer progression stages, and identified that the expression profiles of IRGs could serve as a better classification marker for cancerous tissues of both LUAD and LUSC. We found that the expression changes of IRGs were different between LUAD and LUSC. Cell cycle promoting genes, including KIFs and proteasomes, showed faster up-regulation in LUSC, whereas immune response promoting genes, including MHC molecules and chemokines, were more rapidly repressed in LUSC. Comparative pathway analysis revealed that members of the Toll-like receptor and $\mathrm{T}$ cell receptor signaling pathways exhibited diverged expression changes between LUAD and LUSC, especially at the early cancer stages. Our results revealed the difference of LUAD and LUSC from the immune response point of view, and provided new clues for the differential treatment of LUAD and LUSC.

\section{INTRODUCTION}

Lung cancer is a common and severe disease which ranks the top among cancers worldwide in terms of mortality for both men and women [1,2]. Most of diagnosed lung cancers are malignant epithelial tumors, which could be further classified into small cell lung carcinoma (SCLC) and non-small cell lung carcinoma (NSCLC). NSCLC counts for about $85-90 \%$ of lung cancers, among which the most common subtypes are lung adenocarcinoma (LUAD) and lung squamous cell carcinoma (LUSC) [3]. According to the tumor node metastasis (TNM) taxonomy, both LUAD and LUSC can be classified into four stages, namely stages I, II, III and IV [4]. Stage I refers to the early, non-metastatic stage. Stages II and III usually indicate the intermediate, regional lymphatic metastatic stages, of which stage III has a higher lymphatic metastasis degree than stage II. And stage IV usually represents the late stage with distal metastasis. Each stage can be further divided into A and B sub-stages, of which sub-stage B has higher cancer degree than sub-stage A.

LUAD is at present the most common lung cancer subtype among non-smokers and women, although it has been shown that smoking may increase the risk of LUAD $[5,6]$. By contrast, LUSC is closely associated with smoking, and is more common in men than in women [7]. Generally LUAD grows more slowly with smaller masses than LUSC of the same stage, but LUAD tends to initiate metastasis at the early stages [8]. Pan-cancer studies have shown that the molecular mechanisms of carcinogenesis could be highly heterogeneous between LUAD and LUSC, even in LUAD itself $[3,9,10]$. Consequently, therapies for LUAD are often ineffective for LUSC [11]. 
A number of genes have been reported to be associated with lung cancer, including EGFR, TP53, AKT1, DDR2, FGFR1, KRAS, PTEN, and others [12-18]. Previous studies have shown that the patients' immune system plays a key role in controlling the development of lung cancer [19]. Cancer cells with abnormal mutation could provoke the body's immune responses therefore to be identified and eliminated. It has been shown that animals with deficiency in key immune response related genes are more prone to develop cancers $[20,21]$. On the other hand, cancer cells have also acquired the ability to alter host immune response to facilitate cancer progression [22-25]. Although methods have been developed to diagnose or predict the clinical treatment outcomes of lung cancers basing on the expression profiles of certain immune-response related genes (IRGs) [26-28], thorough comparison of the expression changes of IRGs and their participated pathways/networks during the progression process of LUAD and LUSC is still lacking.

Here, we systematically studied the expression changes of IRGs during the progression process of LUAD and LUSC. Through comparative analysis, we have revealed the expression difference of IRGs between LUAD and LUSC, and identified genes and pathways that may contribute to the faster progression rate of LUSC in comparison to LUAD.

\section{RESULTS}

\section{Immune-response related genes (IRGs) showed systematic expression changes in the LUAD and LUSC samples}

To investigate the expression changes of immuneresponse related genes (IRGs) from the LUAD and LUSC subtype cancer samples, we collected the RNA-Seq data of LUAD and LUSC patients from The Cancer Genome Atlas (TCGA) database. We considered patients with available RNA-seq data from both the tumor and matched normal samples as valid subjects, thus, data from a total of 36 LUAD patients and 32 LUSC patients at different cancer stages were included in this study (Supplementary Table S1 and Supplementary Table S2). We extracted the expression information of IRGs from each sample according to the list of IRGs curated by the Immunology Database and Analysis Portal (IMMPORT) website, which includes genes directly or indirectly involved in immune responses. A total of 6001 IRGs were curated by the IMMPORT dataset, among which, around 4100 were detected in each stage of the LUAD and LUSC samples, respectively (Table 1). Principle component analysis (PCA) showed that the expression profiles of total expressed IRGs could better classify the tumor and normal samples than the profiles of the total expressed genes for both the LUAD and LUSC patients (Figure 1), indicating that the IRGs had undergone systematic and consistent expression changes among the tumor tissues of both LUAD and LUSC.

Using a cutoff threshold of fold change $>2$ and FDR $<0.1$ (with Benjamini-Hochberg multiple testing correction), differentially expressed immune-response related genes (DEIRGs) at each stage of LUAD or LUSC as compared to the corresponding normal tissues were identified by the edgeR software and used in the following analysis (Table 1). Comparative analysis revealed that more than $59 \%$ of the DEIRGs with unidirectional expression changes (see materials and methods) presented in both LUAD and LUSC (Supplementary Figure S1). $\mathrm{GO}$ analysis of the common DEIRGs with unidirectional expression changes revealed that genes involved in multiple immune related processes were down-regulated in both LUAD and LUSC, indicating that the development of both LUAD and LUSC is accompanied by the repression of patient immune responses (Supplementary Figure $\mathrm{S} 2)$. To the contrary, cell cycle and cell division related genes were up-regulated, which was in concert with the fast proliferation feature of cancer cells (Supplementary Figure S2). In addition, more immune related GO terms were enriched among LUSC specifically repressed genes as compared to those of LUAD, which may partially contribute to the faster progression process of LUSC (Supplementary Figure S3).

\section{Expression pattern changes of DEIRGs during cancer progression}

To investigate the expression changes of DEIRGs during the progression of LUAD and LUSC, we classified the expression patterns of DEIRGs using the STEM (short time-series expression miner) software [29]. A total of 7 and 8 significantly enriched expression patterns were identified among DEIRGs of LUAD and LUSC, respectively ( $p$-value $<0.05$, non-parametric clustering algorithm of STEM with Bonferroni correction) (Figure 2 and Supplementary Table S3). Patterns 11, 27, 35 and 42 were identified among DEIRGs of both LUAD and LUSC, whereas patterns 14, 21 and 47 only presented among DEIRGs of LUAD, and patterns 2, 3, 12 and 39 were only detected among DEIRGs of LUSC (Figure 2). By comparing the gene expression level at the most significantly altered cancer stage to that of the normal samples, we roughly divided these patterns into up-regulated and down-regulated expression groups in both LUAD and LUSC (Figure 2). Unexpectedly, only few common genes were identified among the groups with identical patterns in LUAD and LUSC (Supplementary Table S3). Comparative GO analysis revealed that the enriched biological processes among DEIRGs with pattern 42 of LUAD were similar to those among DEIRGs with pattern 35 of LUSC (Supplementary Figure S4), and most of these processes were related to cell proliferation, 
Table 1: No. of expressed immune-response related genes (IRGs) and differentially expressed IRGs of the LUAD and LUSC samples at different cancer stages

\begin{tabular}{|c|c|c|c|c|c|c|c|c|}
\hline \multirow{2}{*}{ Dataset } & \multirow{2}{*}{\multicolumn{2}{|c|}{ Category of IRGs }} & \multirow[t]{2}{*}{ Total } & \multicolumn{5}{|c|}{ Cancer stage } \\
\hline & & & & IA & IB & II & III & IV \\
\hline \multirow{3}{*}{ LUAD } & Expressed & & 4238 & 4158 & 4172 & 4185 & 4166 & 4082 \\
\hline & \multirow{2}{*}{$\begin{array}{l}\text { Differentially } \\
\text { expressed }\end{array}$} & Up & 1336 & 561 & 770 & 896 & 738 & 160 \\
\hline & & Down & 1048 & 551 & 686 & 570 & 746 & 119 \\
\hline \multirow{3}{*}{ LUSC } & Expressed & & 4252 & 4177 & 4201 & 4199 & 4167 & 4015 \\
\hline & \multirow{2}{*}{$\begin{array}{l}\text { Differentially } \\
\text { expressed }\end{array}$} & Up & 1622 & 897 & 1029 & 1094 & 850 & 574 \\
\hline & & Down & 1405 & 678 & 1016 & 950 & 552 & 519 \\
\hline
\end{tabular}

Up, up-regulated genes in tumor samples in comparison to normal samples;

Down, down-regulated genes in tumor samples in comparison to normal samples.
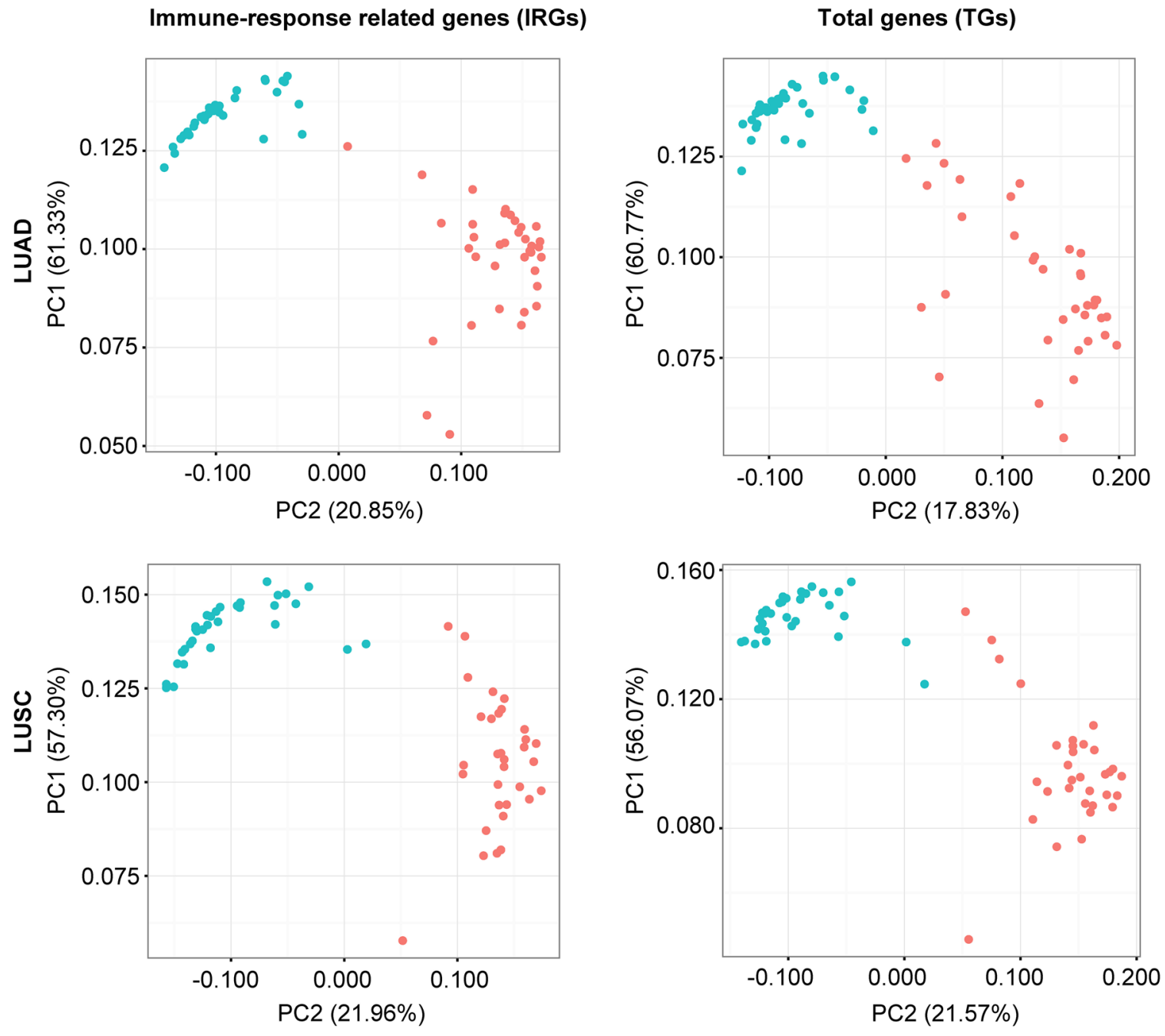

Normal

Tumor

Figure 1: Principle component analysis (PCA) of the expression levels of immune-response related genes (IRGs) and total genes (TGs) among the tumor (red points) and normal (blue points) samples from the LUAD and LUSC patients, respectively. Percentages in parenthesis are the proportion of variability presented by each PCA. 
cell cycle and DNA repair processes (Figure 3A). The expression of genes with pattern 35 reached the peak level at stage IA, whereas the expression of genes with pattern 42 reached the peak level at stage IB. Such earlier activation of cell proliferation and cell cycle related genes in LUSC as compared to LUAD might be one of the causal factors for the faster progression of LUSC. Correspondingly, at stage IA, the expression levels of cell proliferation and cell cycle related genes with pattern 42 of LUAD were generally lower than those with pattern 35 of LUSC (Supplementary Table S4).

To be specific, we found that kinesin superfamily genes $(K I F \mathrm{~s})$ and proteasome complex subunit genes (PSMBs, PSMDs and PSME3) were among genes with pattern 42 of LUAD and pattern 35 of LUSC (Figure 3B and Supplementary Table S4). It has been shown that KIFs play important roles in tumor development and progression due to their crucial functions in regulating cell division as well as intracellular vesicle and organelle transportion [30, 31]. Malfunction of the proteasome complex could also contribute to the pathogenesis of cancer [32-34].

On the other hand, immune response processes related GO terms were significantly enriched among DEIRGs with down-regulated expression patterns, including patterns 11 and 21 of LUAD, and patterns 2, 3, 11, and 12 of LUSC (Figure 3C, Supplementary Figure S5, and Supplementary Table S5). We noticed that $\mathrm{MHC}$ molecules and chemotactic cytokines were overrepresented among the DEIRGs with down-regulated patterns. MHC molecules are crucial for mediating antigen processing and presentation during immune responses [22, $35,36]$. Chemokine can guide the migration of cells, such as directing lymphocytes to the lymph nodes to provoke immune response during immune surveillance [37]. The expression levels of the above mentioned two types of genes at the early stage of LUSC were generally lower than those of LUAD (Figure 3D and Supplementary Table S5), implying their contribution to the faster tumor growth rate of LUSC by escaping the immune surveillance.

\section{Comparative pathway analysis of DEIRGs during LUAD and LUSC progression}

To thoroughly compare the signaling pathways in which DEIRGs participated during LUAD and LUSC progression, we carried out enriched signaling pathway analysis of all DEIRGs with unidirectional expression changes in LUAD and LUSC. Among the identified pathways, Toll-like receptor (TLR) and T cell receptor signaling pathways exhibited synergetic expression differences between LUAD and LUSC. For the TLR pathway, both the upstream TLRs (including TLR2, TLR3, TLR4, TLR5, TLR7 and TLR9) and the downstream effector genes (including CCL3, CCL4 and CCL5 in the complement and coagulation cascade, and $C D 80$ and CD86 with $\mathrm{T}$ cell stimulation functions) all exhibited reduced expression in LUSC as compared with LUAD (Figure 4A). Similarly, genes involved in T cell mediated immune response, such as $C D 3 D, C D 3 E, C D 3 G, C D 247$ in the CD3-TCR complex and downstream effector $Z A P 70$, were more rapidly repressed in LUSC than in LUAD, especially at the early cancer stage (Figure 4B).

\section{DEIRGs with diverged and stage-specific expression patterns}

Next, we searched for DEIRGs exhibited diverged expression changes in LUAD and LUSC. By searching for

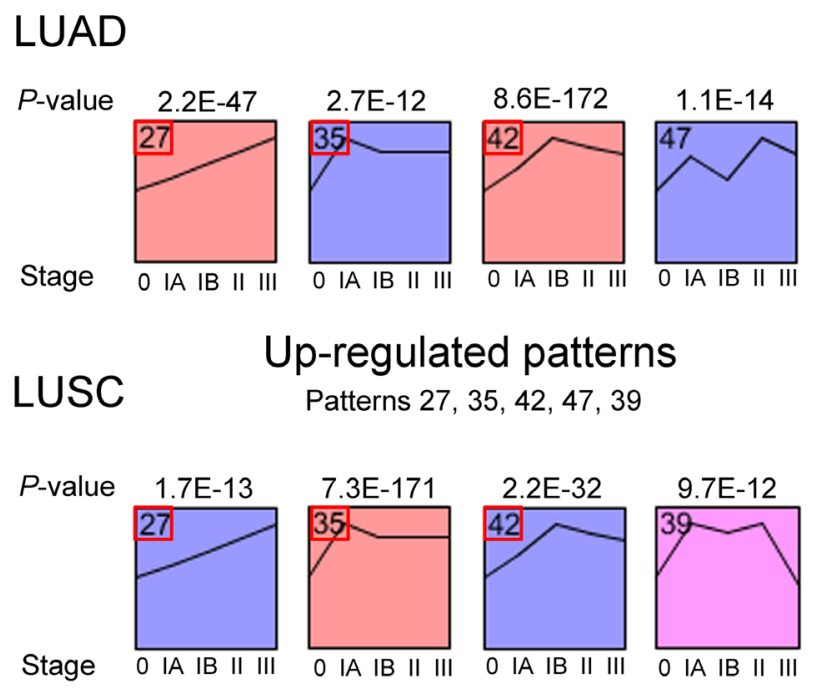

1
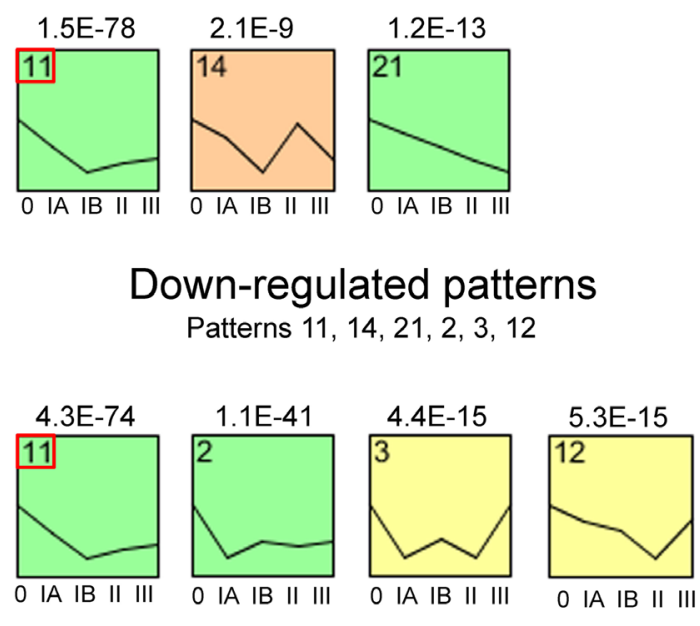

Figure 2: Schematic diagrams of the significantly enriched expression patterns of the differentially expressed immuneresponse related genes (DEIRGs) during LUAD or LUSC progression. Patterns present in both LUAD and LUSC samples are marked with red boxes. 
DEIRGs with unidirectional up-regulation in one cancer subtype whereas with unidirectional repression in the other cancer subtype, we identified 60 DEIRGs being upregulated in LUAD but repressed in LUSC as compared to their corresponding normal tissues (Figure 5A and 5B and Supplementary Table S6), as well as 28 genes being repressed in LUAD but up-regulated in LUSC (Figure 5A and 5D and Supplementary Table S6). In concert with the pathway analysis results, T-cell related processes were the most enriched GO terms among DEIRGs being upregulated in LUAD but repressed in LUSC (Figure 5C). On the other hand, genes being repressed in LUAD but up-regulated in LUSC were enriched of cell adhesion and cell proliferation related functions, which again supported the faster proliferation rate of LUSC (Figure 5E).

We next screened for DEIRGs with specific expression at certain cancer stage (Figure 6A, Supplementary Figure S6 and Supplementary Table S7). Using $>5$ fold up- or down-regulation $($ FDR $<0.1)$ at one cancer stage and without $>2$ fold up- or downregulation $(\mathrm{FDR}<0.1)$ at the other three stages, DEIRGs with either significant up-regulation or repression at any of the examined cancer stages were identified (Figure 6A). Enriched GO terms of these stage-specific genes also
A

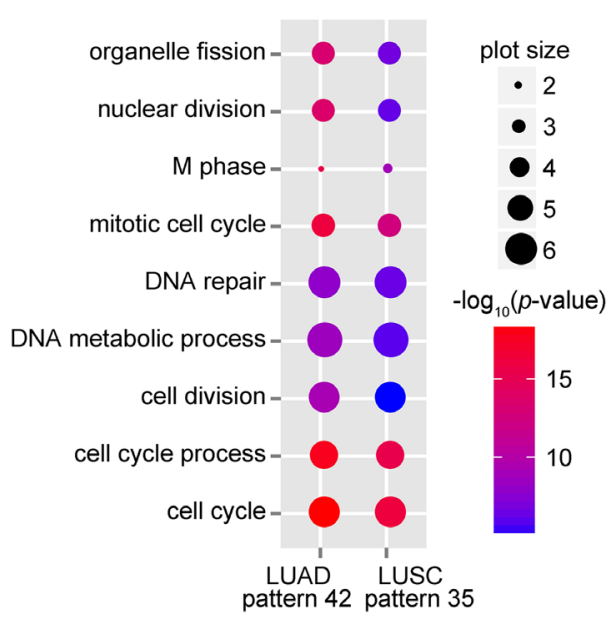

C

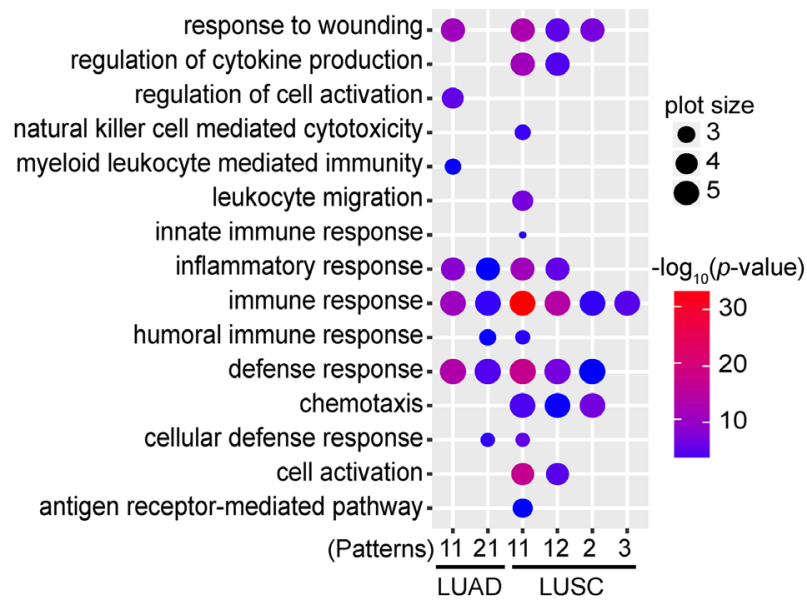

B

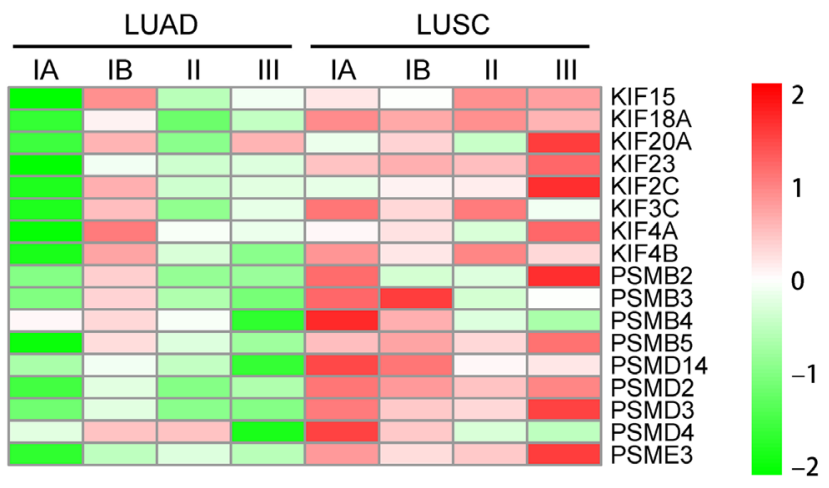

D

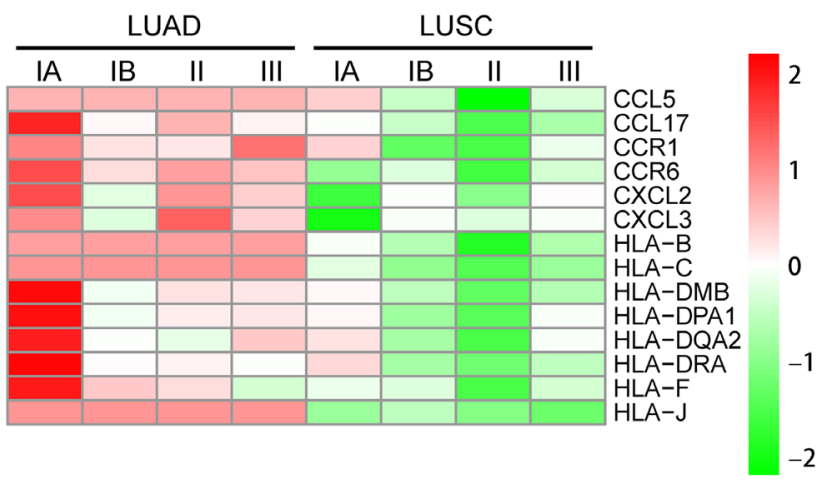

Figure 3: Functional analysis of DEIRGs with significantly enriched expression patterns. A. Commonly enriched GO terms of DEIRGs with pattern 42 of LUAD and pattern 35 of LUSC. Shown are significantly enriched GO terms (FDR $<0.001$, Fisher's exact test) of the biological process category. Dot size represents the frequency of the GO term in the Gene Ontology annotation (GOA) database. Dot color represents the $\log _{10}$-transformed enrichment $p$-value of each GO term. B. Expression changes of the KIFs and proteasome genes during LUAD and LUSC progression. The scaled relative abundances of $\log _{2}$-transformed RPKM ratio (tumor/normal) values are shown in the heatmap. C. Commonly enriched GO terms of DEIRGs with down-regulated expression patterns among LUAD and LUSC samples. Shown are the significantly enriched GO terms (FDR $<0.001$, Fisher's exact test) of the biological process category. Pattern 14 of LUAD is not included as no enriched GO terms were identified among genes of this pattern. D. Expression changes of the MHC and chemokine genes during LUAD and LUSC progression. The scaled relative abundances of $\log _{2}$-transformed RPKM ratio (tumor/normal) values are shown in the heatmap. 
A

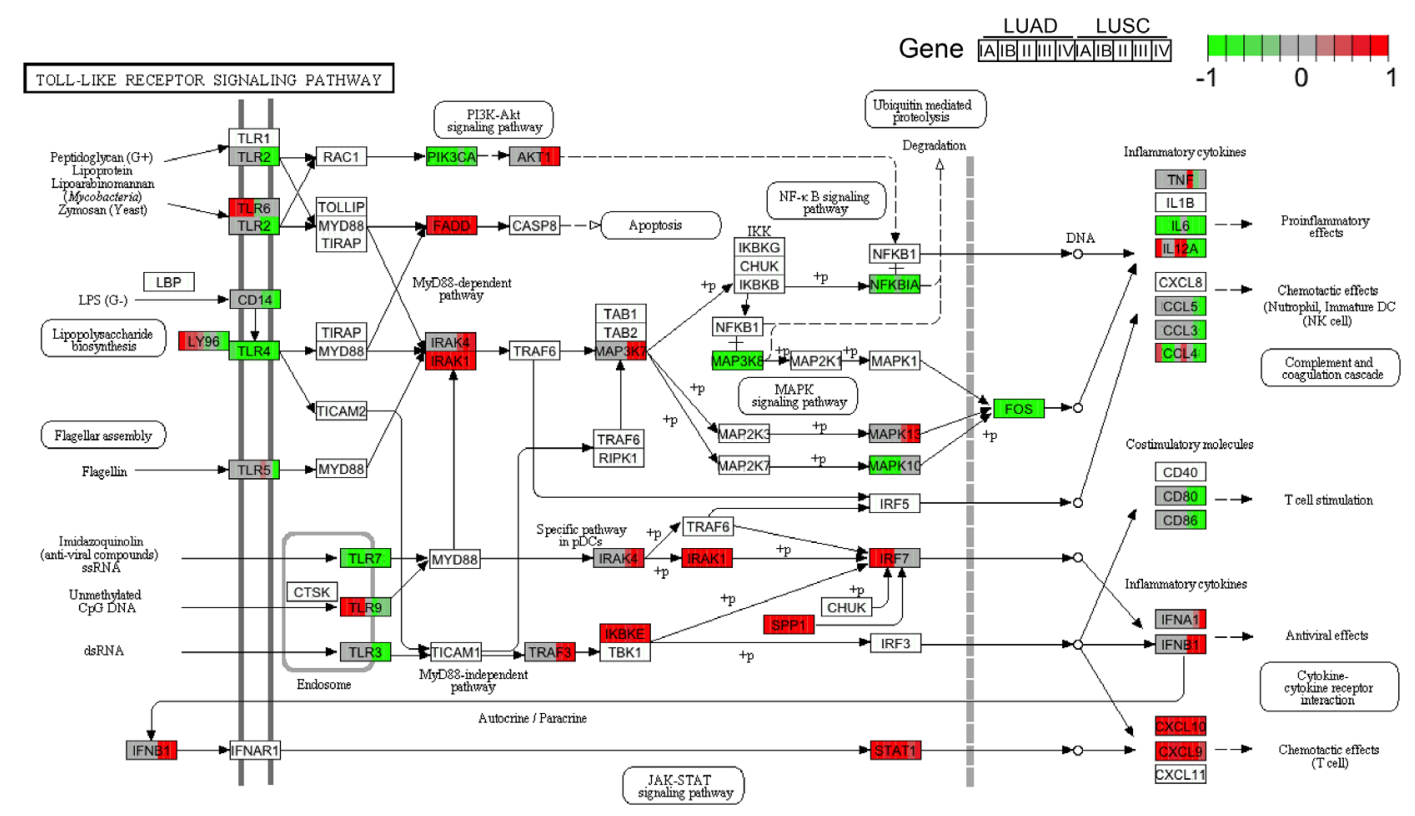

B

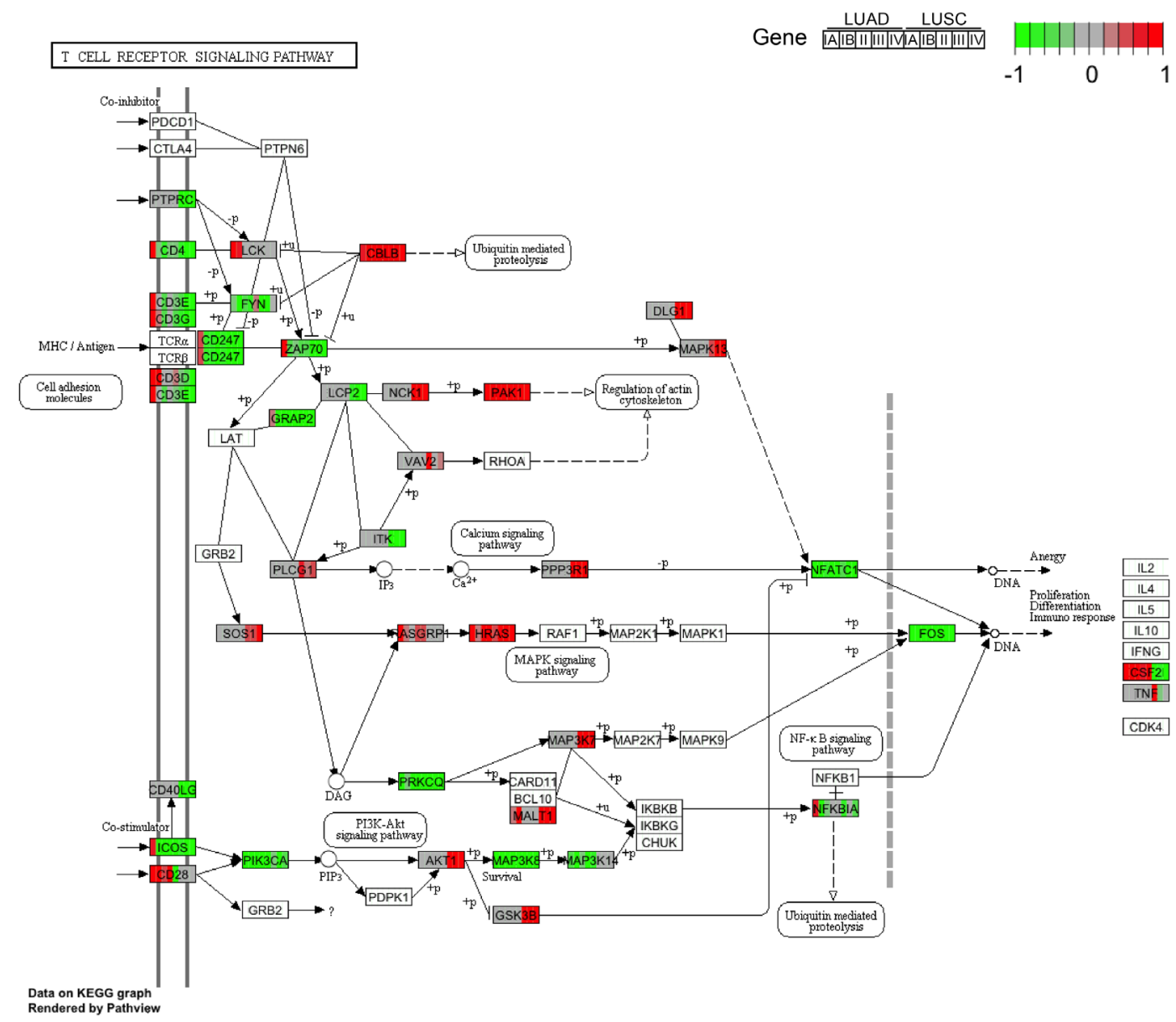

Figure 4: Expression changes of the Toll-like receptor and $T$ cell receptor signaling pathway genes during LUAD and LUSC progression. Each gene box is equally divided into ten pieces, sequentially representing the five stages (IA, IB, II, III, and IV) of LUAD and LUSC. Colors represent the scaled relative abundances of $\log _{2}$-transformed RPKM ratio (tumor/normal) values. A. Expression changes of DEIRGs in the Toll-like receptor signaling pathway. B. Expression changes of DEIRGs in the T cell receptor signaling pathway. 
A

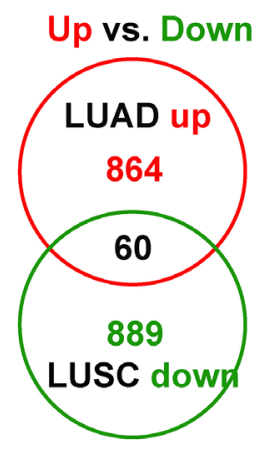

Up vs. Down

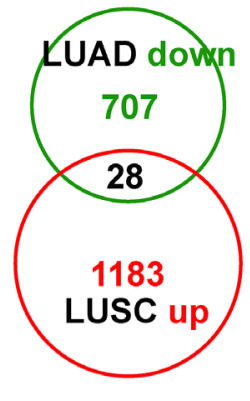

C

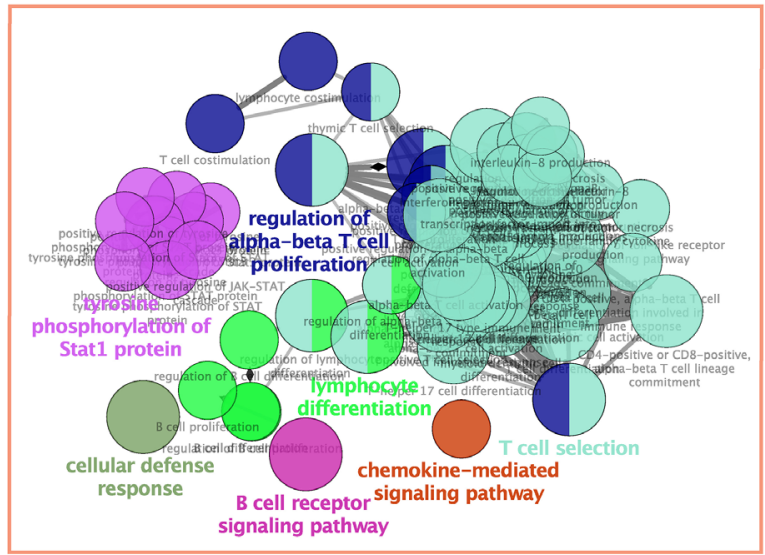

D
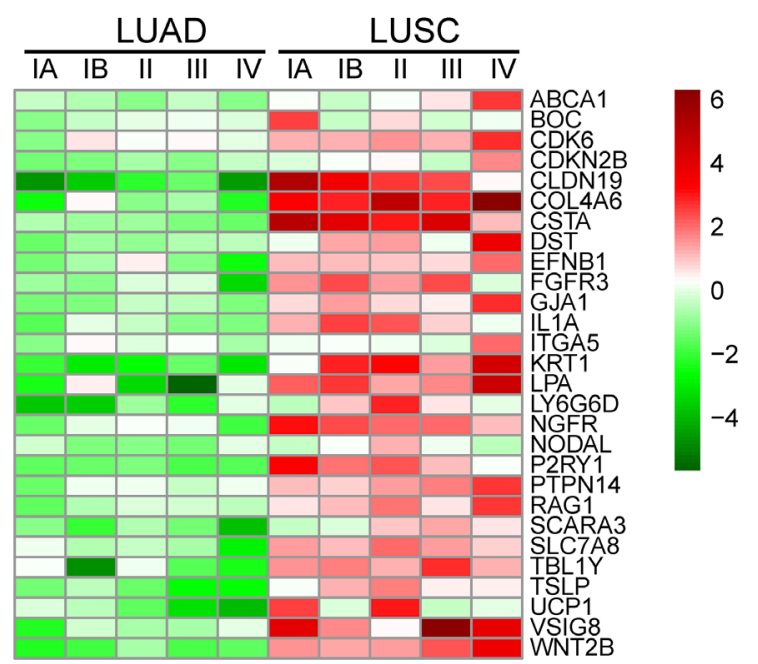

B

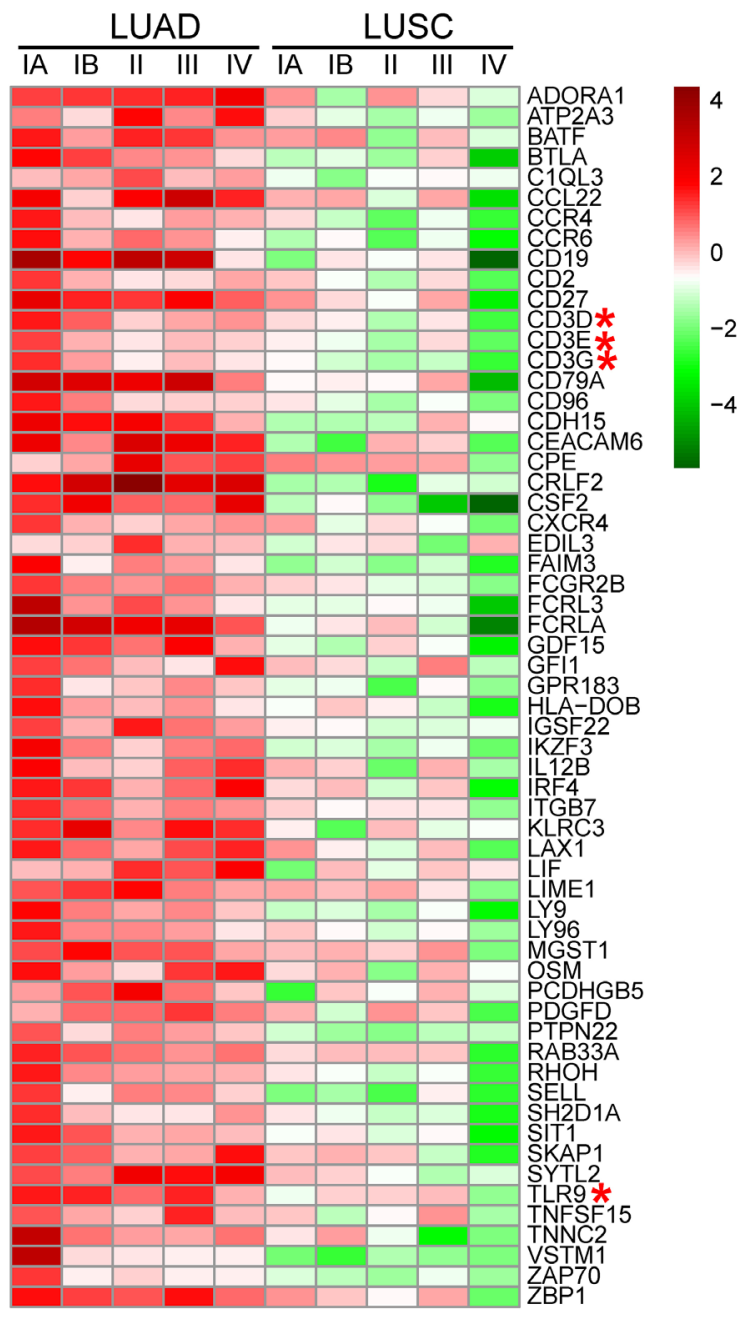

E

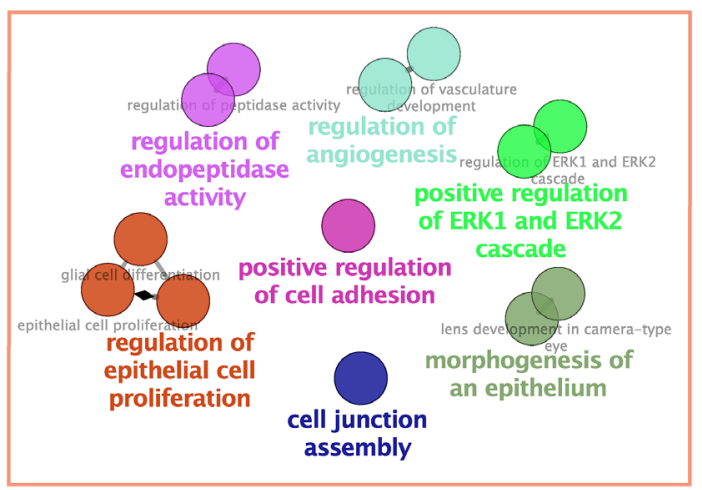

Figure 5: Expression profiles and GO analysis of DEIRGs with diverged changes between LUAD and LUSC. A. Venn diagram analysis of DEIRGs with unidirectional expression changes in LUAD and LUSC. B. Expression profiles of DEIRGs up-regulated in LUAD and down-regulated in LUSC. $\log _{2}$-transformed RPKM ratio (tumor/normal) values are shown in the heatmap. The CD3-TCR complex members and Toll-like receptor were highlighted with red asterisks. C. Enriched GO terms $(p$-value $<0.01$, corrected with Bonferroni step down) of DEIRGs in panel B. The names of processes and their related GO terms are shown in the same colors. Circles are connected according to the hierarchical relationships of GO terms. The sizes of circles are negatively correlated with the enrichment $p$-values of GO terms. D. Expression profiles of DEIRGs down-regulated in LUAD and up-regulated in LUSC. Log 2 -transformed RPKM ratio (tumor/normal) values are shown in the heatmap. E. Enriched GO terms ( $p$-value $<0.05$, corrected with Bonferroni step down) of DEIRGs in panel D. 
differed between LUAD and LUSC, and majority of the GO terms were related to the proliferation and metastasis features of cancer cells (Figure 6B).

\section{DISCUSSION}

Increasing lines of evidence have shown that immune system plays an essential role in controlling cancer progression [19, 38, 39]. Although much efforts have been devoted to identify the causal factors and genes of lung cancers, how are immune-response related genes (IRGs) being regulated in different subtypes of lung cancers is still largely unknown. Here we systematically examined the expression changes of IRGs during the progression process of LUAD and LUSC, and identified that the expression profiles of IRGs could be a better classifier to distinguish LUAD and LUSC from normal tissues.

As the most common subtypes of NSCLC, LUAD and LUSC differ from each other in many aspects [9,
10]. Pathological studies have shown that LUSC grows faster than LUAD [8], but the underlining molecular mechanisms remain unclear. In concert with the clinical features, here we identified that cell cycle and cell proliferation related genes were up-regulated at earlier stage in LUSC than in LUAD, accompanied with the more severe repression of IRGs in LUSC than in LUAD. To be specific, KIFs and proteasome complex unit genes with cell cycle promoting functions had faster upregulated expression in LUSC. On the other hand, MHC molecules and chemokines, which involved in immune response activation, were more rapidly repressed in LUSC. These results could explain the faster progression rate of LUSC as compared with LUAD.

We have also identified Toll-like receptor (TLR) and $\mathrm{T}$ cell receptor signaling pathways to be repressed more severely in LUSC than in LUAD. Reports have shown that the Toll-like receptors play a fundamental role in pathogen recognition and activation of innate
A
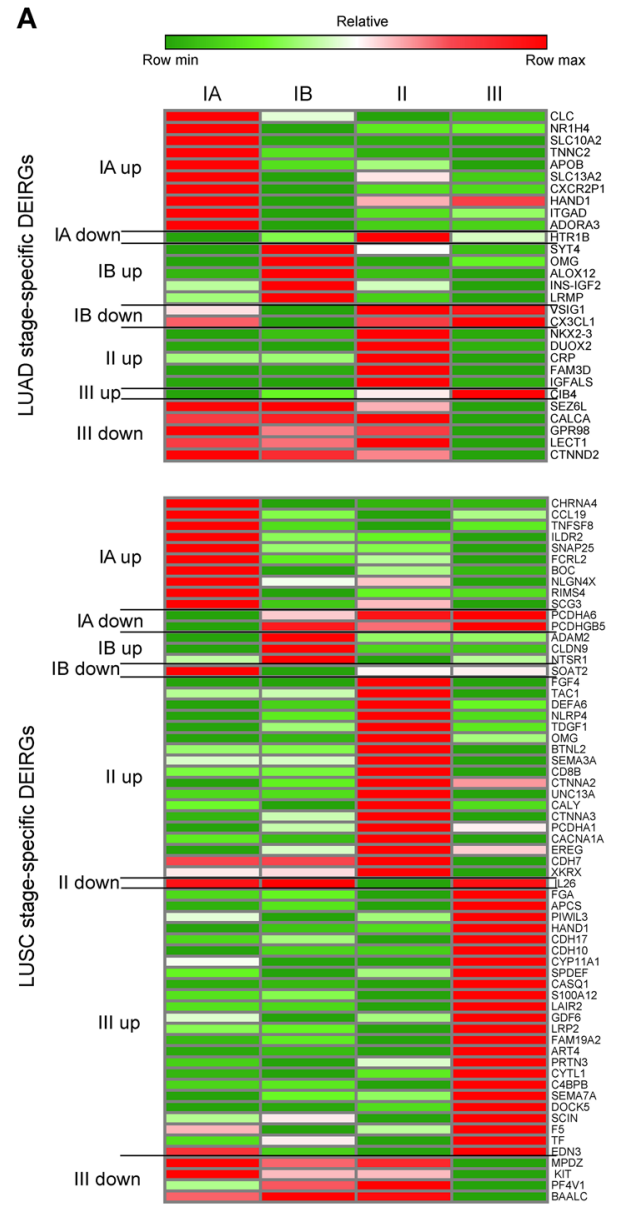

B

LUAD

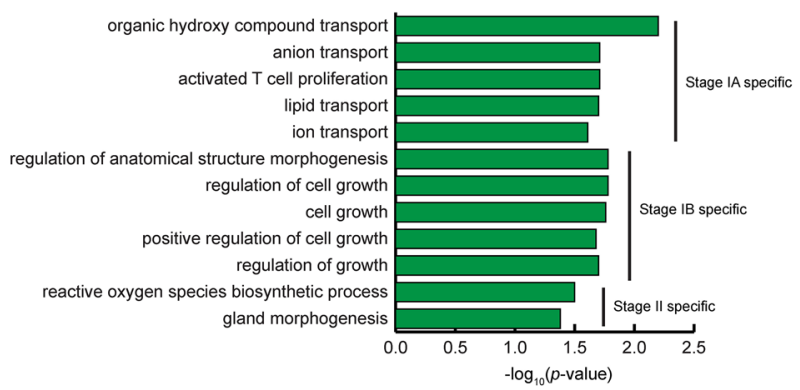

Lusc

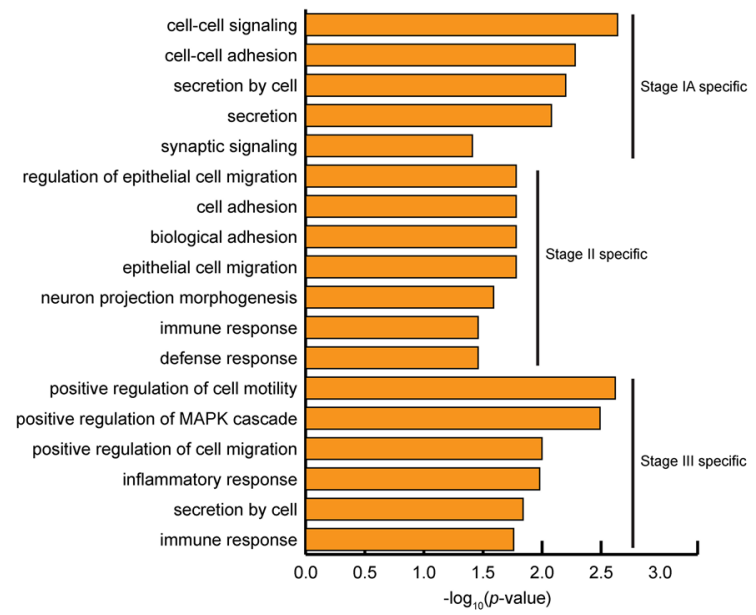

Figure 6: Expression profiles and GO analysis of the stage-specific DEIRGs in LUAD and LUSC. A. Relative expression profiles of the stage-specific DEIRGs. Rows represent stage-specific DEIRGs with their gene symbols to the right, columns represent cancer stages. Up and down indicate the specifically up- and down-regulated DEIRGs at each stage, respectively. Heatmap is generated using the scaled relative abundance of $\log _{2}$-transformed RPKM ratios (tumor/normal). B. Enriched GO terms $(p$-value $<0.05$, Fisher's exact test) of the stage-specific DEIRGs. X-axis represents $\log _{10}$-transformed $p$-values of GO term enrichment and y-axis stands for the enriched GO terms of the biological process category. 
immunity [40-42], and defects of the CD3-TCR complex could also facilitate tumor progression through immune evasion [43]. Thus, we speculated that more rapid repression of $\mathrm{T}$ cell mediated immune response may be correlated with the faster progression rate of LUSC.

In summary, our study revealed the differential expression profiles of IRGs during LUAD and LUSC progression, and identified significantly enriched expression change patterns as well as several other expression features of IRGs during cancer progression process. We discovered that the faster progression rate of LUSC may correlate with the faster activation of cell cycle promoting genes as well as the more rapid repression of immune system response. These results demonstrated the importance of IRGs in regulating the onset and progression of LUAD and LUSC, and may shed lights for the discovery of treatment methods for LUAD and LUSC.

\section{MATERIALS AND METHODS}

\section{Data resources}

The clinical information and RNA-Seq data of LUAD and LUSC patients were downloaded from TCGA database (Data version: 2014_06_14) through TCGAAssembler (Version 1.0.3) (http://health.bsd.uchicago. edu/yji/TCGA-Assembler.htm) [44], using the commands TraverseAllDirectories, DownloadClinicalData, DownloadRNASeqData and the following parameters: assayPlatform "RNASeqV2", dataType "rsem.genes. results" and "rsem.genes.normalized_results".

\section{Sample collection and classification}

RNA-Seq data in the TCGA database satisfying the following criteria were collected and used in this study: 1) samples were collected from the LUAD or LUSC patients; 2) RNA-Seq data of both the tumor and matched normal samples of the same patient should be available; 3) tumor samples had definitive tumor node metastasis (TNM) classification information. Both the LUAD and LUSC samples of selected patients were classified into five stages (IA, IB, II, III, and IV) according to the TNM information assigned by TCGA.

\section{Collection of immune-response related genes}

The list of immune-response related genes (IRGs) was collected from the immunology database and analysis portal (IMMPORT) website (https://immport. niaid.nih.gov) [45], which contains genes either directly or indirectly correlated with the immune system. The expressed IRGs (with normalized counts $\geq 3$ in at least one patient) were selected for further analysis.

\section{Identification of the differentially expressed immune-response related genes (DEIRGs)}

The read count and RPKM matrix tables of IRGs were extracted from classified TCGA RNA-Seq data. IRGs with differential expression (DEIRGs) between the cancer and normal tissues were identified using edgeR (Version 3.10.0) with read count and RPKM matrix tables through the scripts "run_DE_analysis.pl" and "analyze_diff_expr. $\mathrm{pl}^{\prime \prime}$ with default parameters from Trinity software (Version 2.1.1) $[46,47]$. The resulting $p$-values were subjected to Benjamini-Hochberg multiple testing correction to derive FDRs. Only genes with $>2$ fold expression (tumor/ normal) difference and FDR $<0.1$ were considered for further analysis.

\section{Principle component analysis}

The expressed IRGs and total genes (TGs) (with normalized counts $\geq 3$ in at least one patient) were selected to conduct principle component analysis (PCA) by the prcomp function in $\mathrm{R}$ (Version 3.1.0) with the parameter "scale $=\mathrm{T}^{\prime}$.

\section{Selection of DEIRGs with unidirectional expression changes in LUAD or LUSC}

DEIRGs without significant down-regulation at any cancer stage and with $>2$ fold up-regulation (FDR $<0.1)$ at one or more cancer stages were selected as unidirectional up-regulated DEIRGs. Conversely, DEIRGs without significant up-regulation at any cancer stage and with $>2$ fold down-regulation (FDR $<0.1$ ) at one or more cancer stages were selected as unidirectional down-regulated DEIRGs. The Venn diagram and pie chart representing the comparative results of DEIRGs with unidirectional expression changes were plotted using BioVenn, an online tool for comparison and visualization of biological lists (http:// www.cmbi.ru.nl/cdd/biovenn/) [48].

\section{Identification of significantly enriched expression patterns}

Significantly enriched expression patterns of DEIRGs were identified by the STEM (Short Timeseries Expression Miner) software (Version 1.0) [29] using $\log _{2}$-transformed RPKM ratio (tumor/ normal) values as input. Patterns with $p$-value $<0.05$ (non-parametric clustering algorithm of STEM with Bonferroni correction) were identified as enriched patterns. Expression data from stage IV of neither LUAD nor LUSC were included in the analysis due to the limited number of patients (Supplementary Table S2). 


\section{Comparative gene ontology enrichment analysis}

Gene Ontology (GO) enrichment analysis of the common and specific DEIRGs was performed using DAVID Bioinformatics Resources 6.7 (http://david.abcc. ncifcrf.gov/) [49-51] supplemented by the REVIGO visualization toolbox [52]. Plots for enriched GO terms of DEIRGs were generated using the ggplot package (Version 2.1.0) in R [53]. GO enrichment analysis of DEIRGs with diverged expression patterns between LUAD and LUSC was performed by the ClueGO package (Version 2.2.5) of cytoscape (Version 3.3.0) [54, 55].

\section{Pathway enrichment analysis}

Pathway enrichment analysis of DEIRGs with unidirectional expression changes in LUAD and LUSC was performed using EnrichNet (http://www.enrichnet. org/) [56-58]. Enriched genes in selected pathways were shown using the Pathview package (Version 1.8.0) in $\mathrm{R}$ $[59,60]$.

\section{Screen for DEIRGs with diverged or stage- specific expression patterns}

DEIRGs with diverged expression patterns were obtained through comparing the unidirectional upregulated genes in LUAD with the unidirectional downregulated genes in LUSC, and vice versa.

DEIRGs with $>5$ fold up-regulation $(\mathrm{FDR}<0.1)$ at one cancer stage and without significant up-regulation at other cancer stages were selected as up-regulated stagespecific DEIRGs. Conversely, DEIRGs with $>5$ fold down-regulation $(\mathrm{FDR}<0.1)$ at one cancer stage and without significant down-regulation at other cancer stages were selected as down-regulated stage-specific DEIRGs. The heatmaps were generated using pheatmap package in R or GENE-E software (Version 2.1.134) (http://www. broadinstitute.org/cancer/software/GENE-E/). Expression data from stage IV of neither LUAD nor LUSC were included in the analysis due to the limited number of patients.

\section{ACKNOWLEDGMENTS}

This result here is in whole based upon the data generated by TCGA Research Network: http:// cancergenome.nih.gov/. We gratefully acknowledge contributions from the TCGA Research Network and the specimen donors.

\section{CONFLICTS OF INTEREST}

The authors declare no conflicts of interest.

\section{GRANT SUPPORT}

This work was supported by the National Natural Science Foundation of China (NSFC No. 31271349, 81273275), National High-tech R\&D Program of China (863 Program) 2012AA020409, the Research and Development Foundation of Science and Technology of Shenzhen (JCYJ20150525092941055).

\section{Author contributions}

M.C., L.X. and X.-J. W. designed the project. M.C. and X.L. performed bioinformatic analysis. M.C., X.-J. W. and L.X. wrote the manuscript. All authors discussed the results and revised the manuscript.

\section{REFERENCES}

1. Siegel RL, Miller KD, Jemal A. Cancer statistics, 2015. CA: a cancer journal for clinicians. 2015; 65:5-29.

2. Chen W, Zheng R, Baade PD, Zhang S, Zeng H, Bray F, Jemal A, Yu XQ, He J. Cancer statistics in China, 2015. CA: a cancer journal for clinicians. 2016; 66:115-132.

3. Chang JTH, Lee YM, Huang RS. The impact of the Cancer Genome Atlas on lung cancer. Translational Research. 2015; 166:568-585.

4. Detterbeck FC, Boffa DJ, Tanoue LT. The New Lung Cancer Staging System. Chest. 2009; 136:260-271.

5. Subramanian J, Govindan R. Lung cancer in never smokers: a review. Journal of clinical oncology. 2007; 25:561-570.

6. Couraud S, Zalcman G, Milleron B, Morin F, Souquet PJ. Lung cancer in never smokers--a review. European journal of cancer (Oxford, England : 1990). 2012; 48:1299-1311.

7. Kenfield SA, Wei EK, Stampfer MJ, Rosner BA, Colditz GA. Comparison of aspects of smoking among the four histological types of lung cancer. Tobacco Control. 2008; 17:198-204.

8. Aster JC, Abbas AK, Robbins SL, Kumar V. (2013). Robbins basic pathology (Ninth edition). Philadelphia, PA: Elsevier Saunders.

9. Hammerman PS, Lawrence MS, Voet D, Jing R, Cibulskis K, Sivachenko A, Stojanov P, McKenna A, Lander ES, Gabriel S, Getz G, Sougnez C, Imielinski M, et al. Comprehensive genomic characterization of squamous cell lung cancers. Nature. 2012; 489:519-525.

10. Collisson EA, Campbell JD, Brooks AN, Berger AH, Lee W, Chmielecki J, Beer DG, Cope L, Creighton CJ, Danilova L, Ding L, Getz G, Hammerman PS, et al. Comprehensive molecular profiling of lung adenocarcinoma. Nature. 2014; 511:543-550.

11. Rekhtman N, Paik PK, Arcila ME, Tafe LJ, Oxnard GR, Moreira AL, Travis WD, Zakowski MF, Kris MG, Ladanyi M. Clarifying the spectrum of driver oncogene mutations in biomarker-verified squamous carcinoma of lung: lack of 
EGFR/KRAS and presence of PIK3CA/AKT1 mutations. Clinical Cancer Research. 2012; 18:1167-1176.

12. Bleeker FE, Felicioni L, Buttitta F, Lamba S, Cardone L, Rodolfo M, Scarpa A, Leenstra S, Frattini M, Barbareschi M, Grammastro MD, Sciarrotta MG, Zanon C, et al. AKT1(E17K) in human solid tumours. Oncogene. 2008; 27:5648-5650.

13. Hammerman PS, Sos ML, Ramos AH, Xu C, Dutt A, Zhou W, Brace LE, Woods BA, Lin W, Zhang J, Deng X, Lim $\mathrm{SM}$, Heynck S, et al. Mutations in the DDR2 kinase gene identify a novel therapeutic target in squamous cell lung cancer. Cancer discovery. 2011; 1:78-89.

14. Dutt A, Ramos AH, Hammerman PS, Mermel C, Cho J, Sharifnia T, Chande A, Tanaka KE, Stransky N, Greulich H, Gray NS, Meyerson M. Inhibitor-sensitive FGFR1 amplification in human non-small cell lung cancer. PloS one. 2011; 6:e20351.

15. Brose MS, Volpe P, Feldman M, Kumar M, Rishi I, Gerrero R, Einhorn E, Herlyn M, Minna J, Nicholson A, Roth JA, Albelda SM, Davies H, et al. BRAF and RAS mutations in human lung cancer and melanoma. Cancer research. 2002; 62:6997-7000.

16. Bean J, Brennan C, Shih JY, Riely G, Viale A, Wang L, Chitale D, Motoi N, Szoke J, Broderick S, Balak M, Chang WC, Yu CJ, et al. MET amplification occurs with or without T790M mutations in EGFR mutant lung tumors with acquired resistance to gefitinib or erlotinib. Proceedings of the National Academy of Sciences of the United States of America. 2007; 104:20932-20937.

17. Kawano O, Sasaki H, Endo K, Suzuki E, Haneda H, Yukiue H, Kobayashi Y, Yano M, Fujii Y. PIK3CA mutation status in Japanese lung cancer patients. Lung cancer (Amsterdam, Netherlands). 2006; 54:209-215.

18. Jin G, Kim MJ, Jeon HS, Choi JE, Kim DS, Lee EB, Cha SI, Yoon GS, Kim CH, Jung TH, Park JY. PTEN mutations and relationship to EGFR, ERBB2, KRAS, and TP53 mutations in non-small cell lung cancers. Lung cancer (Amsterdam, Netherlands). 2010; 69:279-283.

19. Mazzoccoli G, Grilli M, Carughi S, Puzzolante F, De Cata A, La Viola M, Giuliani A, Urbano N, Tarquini R, Perfetto F. Immune system alterations in lung cancer patients. International Journal of Immunopathology and Pharmacology. 2003; 16:167-174.

20. Ichiki Y, Takenoyama M, Mizukami M, So T, Sugaya M, Yasuda M, So T, Hanagiri T, Sugio K, Yasumoto K. Simultaneous cellular and humoral immune response against mutated p53 in a patient with lung cancer. Journal of Immunology. 2004; 172:4844-4850.

21. Jassar AS, Suzuki E, Kapoor V, Sun J, Silverberg MB, Cheung LM, Burdick MD, Strieter RM, Ching LM, Kaiser LR, Albelda SM. Activation of tumorassociated macrophages by the vascular disrupting agent 5,6-dimethylxanthenone-4-acetic acid induces an effective CD8(+) T-cell-mediated antitumor immune response in murine models of lung cancer and mesothelioma. Cancer Research. 2005; 65:11752-11761.

22. So T, Takenoyama M, Mizukami M, Ichiki Y, Sugaya M, Hanagiri T, Sugio K, Yasumoto K. Haplotype loss of HLA class I antigen as an escape mechanism from immune attack in lung cancer. Cancer Research. 2005; 65:5945-5952.

23. He W, Liu Q, Wang L, Chen W, Li N, Cao X. TLR4 signaling promotes immune escape of human lung cancer cells by inducing immunosuppressive cytokines and apoptosis resistance. Molecular Immunology. 2007; 44:2850-2859.

24. Dougan M, Li D, Neuberg D, Mihm M, Googe P, Wong $\mathrm{K}-\mathrm{K}$, Dranoff G. A dual role for the immune response in a mouse model of inflammation-associated lung cancer. Journal of Clinical Investigation. 2011; 121:2436-2446.

25. Bremnes RM, Al-Shibli K, Donnem T, Sirera R, Al-Saad S, Andersen S, Stenvold H, Camps C, Busund L-T. The role of tumor-infiltrating immune cells and chronic inflammation at the tumor site on cancer development, progression, and prognosis emphasis on non-small cell lung cancer. Journal of Thoracic Oncology. 2011; 6:824-833.

26. Roepman P, Jassem J, Smit EF, Muley T, Niklinski J, van de Velde T, Witteveen AT, Rzyman W, Floore A, Burgers S, Giaccone G, Meister M, Dienemann H, et al. An immune response enriched 72-gene prognostic profile for early-stage non-small-cell lung cancer. Clinical Cancer Research. 2009; 15:284-290.

27. Suzuki K, Kachala SS, Kadota K, Shen R, Mo Q, Beer DG, Rusch VW, Travis WD, Adusumilli PS. Prognostic immune markers in non-small cell lung cancer. Clinical Cancer Research. 2011; 17:5247-5256.

28. Holt GE, Disis ML. Immune modulation as a therapeutic strategy for non-small-cell lung cancer. Clinical Lung Cancer. 2008; 9:S13-S19.

29. Ernst J, Bar-Joseph Z. STEM: a tool for the analysis of short time series gene expression data. Bmc Bioinformatics. 2006; 7.

30. Rath O, Kozielski F. Kinesins and cancer. Nature Reviews Cancer. 2012; 12:527-539.

31. Yu Y, Feng Y-M. The role of kinesin family proteins in tumorigenesis and progression. Cancer. 2010; 116:5150-5160.

32. Adams J. Potential for proteasome inhibition in the treatment of cancer. Drug Discovery Today. 2003; 8:307-315.

33. Adams J. The proteasome: A suitable antineoplastic target. Nature Reviews Cancer. 2004; 4:349-360.

34. Zhang Z, Zhang R. Proteasome activator PA28 gamma regulates $\mathrm{p} 53$ by enhancing its MDM2-mediated degradation. Embo Journal. 2008; 27:852-864.

35. Benacerraf B. Role of MHC gene products in immune regulation. Science. 1981; 212:1229-1238. 
36. Garcia-Lora A, Algarra I, Garrido F. MHC class I antigens, immune surveillance, and tumor immune escape. Journal of Cellular Physiology. 2003; 195:346-355.

37. Moser B, Wolf M, Walz A, Loetscher P. Chemokines: multiple levels of leukocyte migration control. Trends in Immunology. 2004; 25:75-84.

38. Brahmer JR. Harnessing the immune system for the treatment of non-small-cell lung cancer. Journal of Clinical Oncology. 2013; 31:1021-1028.

39. Chen C, Shen Y, Qu Q-X, Chen X-Q, Zhang X-G, Huang $\mathrm{J}-\mathrm{A}$. Induced expression of B7-H3 on the lung cancer cells and macrophages suppresses T-cell mediating anti-tumor immune response. Experimental Cell Research. 2013; 319:96-102.

40. Akira S, Takeda K. Toll-like receptor signalling. Nature Reviews Immunology. 2004; 4:499-511.

41. Iwasaki A, Medzhitov R. Toll-like receptor control of the adaptive immune responses. Nature Immunology. 2004; 5:987-995.

42. Krieg AM. Toll-like receptor 9 (TLR9) agonists in the treatment of cancer. Oncogene. 2008; 27:161-167.

43. Kuang DM, Zhao Q, Xu J, Yun JP, Wu C, Zheng L. Tumor-educated tolerogenic dendritic cells induce CD3ع down-regulation and apoptosis of $\mathrm{T}$ cells through oxygendependent pathways. Journal of immunology (Baltimore, Md : 1950). 2008; 181:3089-3098.

44. Zhu Y, Qiu P, Ji Y. TCGA-Assembler: open-source software for retrieving and processing TCGA data. Nature Methods. 2014; 11:599-600.

45. Bhattacharya S, Andorf S, Gomes L, Dunn P, Schaefer H, Pontius J, Berger P, Desborough V, Smith T, Campbell J, Thomson E, Monteiro R, Guimaraes P, et al. ImmPort: disseminating data to the public for the future of immunology. Immunologic Research. 2014; 58:234-239.

46. Robinson MD, McCarthy DJ, Smyth GK. edgeR: a Bioconductor package for differential expression analysis of digital gene expression data. Bioinformatics. 2010; 26:139-140.

47. Grabherr MG, Haas BJ, Yassour M, Levin JZ, Thompson DA, Amit I, Adiconis X, Fan L, Raychowdhury R, Zeng Q, Chen Z, Mauceli E, Hacohen N, et al. Full-length transcriptome assembly from RNA-Seq data without a reference genome. Nat Biotechnol. 2011; 29:644-652.

48. Hulsen T, de Vlieg J, Alkema W. BioVenn - a web application for the comparison and visualization of biological lists using area-proportional Venn diagrams. Bmc Genomics. 2008; 9.
49. Huang DW, Sherman BT, Lempicki RA. Systematic and integrative analysis of large gene lists using DAVID bioinformatics resources. Nature Protocols. 2009; 4:44-57.

50. Huang DW, Sherman BT, Lempicki RA. Bioinformatics enrichment tools: paths toward the comprehensive functional analysis of large gene lists. Nucleic Acids Research. 2009; 37:1-13.

51. Harris MA, Clark J, Ireland A, Lomax J, Ashburner M, Foulger R, Eilbeck K, Lewis S, Marshall B, Mungall C, Richter J, Rubin GM, Blake JA, et al. The Gene Ontology (GO) database and informatics resource. Nucleic Acids Research. 2004; 32:D258-D261.

52. Supek F, Bosnjak M, Skunca N, Smuc T. REVIGO summarizes and visualizes long lists of gene ontology terms. Plos One. 2011; 6.

53. Ginestet C. ggplot2: Elegant graphics for data analysis. Journal of the Royal Statistical Society Series a-Statistics in Society. 2011; 174:245-245.

54. Bindea G, Mlecnik B, Hackl H, Charoentong P, Tosolini M, Kirilovsky A, Fridman WH, Pages F, Trajanoski Z, Galon J. ClueGO: a Cytoscape plug-in to decipher functionally grouped gene ontology and pathway annotation networks. Bioinformatics. 2009; 25:1091-1093.

55. Shannon P, Markiel A, Ozier O, Baliga NS, Wang JT, Ramage D, Amin N, Schwikowski B, Ideker T. Cytoscape: A software environment for integrated models of biomolecular interaction networks. Genome Research. 2003; 13:2498-2504.

56. Glaab E, Baudot A, Krasnogor N, Schneider R, Valencia A. EnrichNet: network-based gene set enrichment analysis. Bioinformatics. 2012; 28:I451-I457.

57. Stelzl U, Worm U, Lalowski M, Haenig C, Brembeck FH, Goehler H, Stroedicke M, Zenkner M, Schoenherr A, Koeppen S, Timm J, Mintzlaff S, Abraham C, et al. A human protein-protein interaction network: A resource for annotating the proteome. Cell. 2005; 122:957-968.

58. Szklarczyk D, Franceschini A, Wyder S, Forslund K, Heller D, Huerta-Cepas J, Simonovic M, Roth A, Santos A, Tsafou KP, Kuhn M, Bork P, Jensen LJ, et al. STRING v10: protein-protein interaction networks, integrated over the tree of life. Nucleic Acids Research. 2015; 43:D447-D452.

59. Luo WJ, Brouwer C. Pathview: an R/Bioconductor package for pathway-based data integration and visualization. Bioinformatics. 2013; 29:1830-1831.

60. Kanehisa M, Goto S. KEGG: kyoto encyclopedia of genes and genomes. Nucleic Acids Research. 2000; 28:27-30. 